\title{
Entropy Based Target Identification in Synthetic Aperture Radar Polarimetry
}

\author{
Plasin Francis Dias, R. M. Banakar
}

\begin{abstract}
Synthetic aperture radar is used for polarimetric target identification. It is most prominent imaging radar. This radar covers the widest ranges of earth crust with high resolution images. It captures images day and night. It is suitable for any seasonal weather conditions. The polarization data contains information, on scattering mechanism related to different objects. The objects are land, ocean, glaceries, snow and dense forest which are natural distributed targets. By the use of scattering mechanism the different objects are classified. Scattering mechanism is measured by scattering elements of the matrix. The full polarization of synthetic aperture radar data classifies the obtained image. This paper analyses an entropy based target identification related to synthetic aperture radar polarimetry. The method is also the outcome of Eigen decomposition analysis. The paper also gives broader view of identification of target using physical property and analytical model. The method is helpful for system level design and scattering process considerations.
\end{abstract}

Keywords-Synthetic Aperture Radar, Polarimetry, Eigen, Decomposition, Entropy, Coherency matrix

\section{INTRODUCTION}

To identify targets efficiently advanced airborne and space borne Synthetic Aperture Radars (SAR) are designed and developed. In this radar the two platforms that is sensor and target region both are in relative motion. The SAR processing unit is mounted on a moving system basically aircraft or space craft. The target is covered by single beam by the radar antenna. The target area is continuously illuminated with pulses of radio waves, at wave lengths meters to $\mathrm{mm}$. The obtained synthetic radar image can be classified by the full polarization information of the synthetic aperture radar data. The accessing of full polarization is the basic technique behind the SAR polarimetry. It is a method to obtain the target details which is basically based on amplitude and phase related information. The term full polarization refers to the four main channels used in quad (full) polarimetry. They are polarized in four directions namely horizontal -horizontal $(\mathrm{HH})$, horizontal -vertical (HV), vertical horizontal (VH) and vertical - vertical (VV) directions.

The scattering mechanism describes the physical details of the target. The physical details of the target are referenced to its dielectric constant and orientation. It is obtained through various scattering mechanisms. The radar SAR sensor transmits continuously the electromagnetic wave towards the target. In response to incident wave the various echo waveforms are received by the receiving antenna. At several different antenna positions, the target is detected with coherency. The received signals are called as echo signals. The scattering matrix representing the information of echo signals has the four basic elements $\mathrm{S}_{\mathrm{HH}}, \mathrm{S}_{\mathrm{HV}}, \mathrm{S}_{\mathrm{VH}}, \mathrm{S}_{\mathrm{VV}}$.

Revised Manuscript Received on December 16, 2019

* Correspondence Author

Plasin Francis Dias ${ }^{1}$, Electronics and Communication Engineering, KLS VDIT, Haliyal, India. Email: plasindias@gmail.com

R. M. Banakar*, Electronics and Communication Engineering, BVBCET, Hubli, India. Email: banakar@bvb.edu
The properties of individual objects of the earth surface are characterized by their own scattering nature. This scattering nature is represented mathematically as the scattering matrix for each pixel of the target image. The received signal obtained by SAR sensor adds for criteria called resolution cell. It is basically the resultant criteria of many distributed targets. These targets are distributed in spatial form. Every individual or target is represented by different types of scattering mechanisms. The various scattering mechanisms involved are surface scattering also known as single bounce scattering. The other mechanisms are typically known as double bounce, volume and helix scattering. These are obtained by the natural targets and manmade targets. The physical property surface roughness and its materialistic property namely dielectric constant decide which type of scattering mechanisms occurs. The feature called coherent is basic element criteria of the scattering matrix. It means that the terms in off diagonal element of scattering matrix that is $\mathrm{S}_{\mathrm{HV}}$ and $\mathrm{S}_{\mathrm{VH}}$ are equal. Scattering matrix normally gives the detail idea of the working pattern of radar system, in hitting the particular target on the earth surface. It also describes the behavior of the targets on the earth surface for the feature called scatter. This represents the electromagnetic energy returned back towards the radar sensor.

While evaluating the image information the dimension of the $\mathrm{S}$ matrix is considered as $2 * 2$. It is hence considered for every pixel of an image. This concept is illustrated by the authors Fawag Ulaby and Ehsrlon Elactic in contribution toward their paper radar polarimetry for geo science applications [2]. The responses obtained by two same points always have minimum observable spacing which contributes for resolution. This is again applied to width of one more response other than previous two. A weaker response among them basically requires larger separation for the detection of the target. Each resolution cell is represented by two pixels. Pixel spacing in SAR imagery is considered for some standard scale. It is normally taken as multiple or division of $100 \mathrm{mts}$. For ex. ERS -1 data has resolution of $28 \mathrm{mts}$. In range and azimuth it is delivered with $12.5 \mathrm{mt}$ pixel spacing. In SAR image, the direction of aircraft or space craft is called azimuth direction. The imaging direction is called range direction. SAR processing involves range focusing and azimuth focusing. Range focusing involves de-chirping of received echoes. Azimuth focusing relies on Doppler information produced by target. The difference in dimension of pixel of signal related to SAR image exists to the resolution cell during the data acquisition. It is due to variation of range resolution angle. So pixel resampling is carried out with uniform grid.

The information obtained by SAR polarimetry refers to the data of polarization state of received signals. It is represented for every resolution element or pixel. The behavior of the target is a mathematical representation which is modeled in terms of scattering matrix.

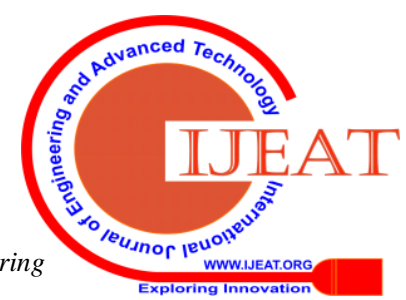




\section{Entropy Based Target Identification in Synthetic Aperture Radar Polarimetry}

This is basically the information of electromagnetic wave reflected by the objects. Current airborne systems have an azimuth resolution of $1 \mathrm{~m}$ to $0.5 \mathrm{~m}$. The resolution of image gives the separation between closest surfaces, which is basically resolved in the final image. The authors in [14] [16] explain with illustrative figures the concept of SAR resolution.

. The recent advances in SAR polarimetry features the fundamentals of processing SAR data using mathematical model in an organized way [7]. Polarimetry is the study of measurement and investigation method of the polarization pattern of transverse electromagnetic waves. These EM waves are also known as radio or light waves. Polarimetry is carried out on such electromagnetic waves, which are already are reflected or refracted or diffracted waves. These reflection or refraction or diffraction will occur through some material. This change in features will characterize the particular object. Depending on the data received by acquisition method various processing techniques are followed. The processing of SAR data is limited by processing of intensity from target image acquired. The Interferometric processing involves intensity and interferometric phase processing. In interferometry phase and intensity are the main parameters. The target image is processed using various techniques. In interferometry only one image is acquired featuring phase and intensity. In polarimetry the data acquisition is done using different polarization techniques.

Polarimetric processing involves intensity and polarimetric phase processing. In [1][15] the authors illustrate the fundamental aspects of polarization. Polarization is the method of alignment of electric and magnetic field. These fields are the elementary components of Electromagnetic waves. Although these two fields illustrate the characterization of EM waves, its behavior can be solely done by using the electric field. This is possible because the electric and magnetic field has distinct mathematical relationship. Hence the entire EM wave can be investigated only by knowing the electric field vector. The electric field is represented as a vector. This vector has a length which distinctly represents the amplitude of the electromagnetic wave. The vector rotation rate gives the frequency of the wave. The vector movement can be in different directions namely linear, elliptical and circular. Polarimetric interferometric processing involves intensity, interferometric and polarimetric phase processing which is a new and advanced technique.

The radar frequency or wavelength, polarization and incidence angles affect the scattering mechanisms. If surface is found smooth the existence of depolarization is considered as nil. It implies $\mathrm{HV}$ or VH back scatter is not present. If the surface is rough then there will be some depolarization existing. Hence $\mathrm{HV}$ or $\mathrm{VH}$ backscatter exists. For volume scattering such as forest and vegetation high depolarization is found. Thus one can categorize various objects based on the scattering mechanisms. There is peculiar relation exist between scattering mechanisms, scattering matrix and various decomposition techniques involved in the SAR polarimetry. The section II highlights the importance of coherency matrices in the evaluation of Eigen decomposition for entropy based target identification.

\section{CO VARIANCE AND COHERENCY MATRICES}

The scattering properties of a target are basically described by various forms of mathematical representation. The role precisely works when it comes to the power domain criteria. So the SAR image is identified with various matrices known as Coherency and Covariance matrix. The other forms of matrix are Muller, Stokes and Scattering matrix. The reflected wave from the target has different polarization in relation to incident wave of the radar sensor. The scattering matrix consists of 4 basic elements $S_{h h}, S_{h v}, S_{v h}$ and $S_{v v}$ which are complex in values. The response of the target is function of incident electromagnetic wave as well as back scattered polarizations. The scattering power can be determined by 4 polarization variables $\mathrm{HH}, \mathrm{HV}, \mathrm{VH}$ and $\mathrm{VV}$, the incident angle, elipticity angle, orientation and reflected angles.

The interaction of electromagnetic wave with objects forms scattering mechanism related to objects. Polarimetric information of given pixel is representation of scattering mechanisms. To interpret this scattering mechanism and objects properties polarimetric decomposition models are developed. The two vectors called covariance vector and coherency vector plays the major role in forming the two specific matrices called as covariance matrix and coherency matrix. The covariance vector is represented by symbol $k_{C}$. It is the vector representation of scattering matrix. It also forms the basis for linear decomposition technique of SAR polarimetry. The self multiplication of covariance vector and its conjugate transpose will lead to the formation of covariance matrix. The coherency vector is represented by symbol. This vector is basis for Pauli decomposition analysis. Kpe elements of coherency vector describes the physical features of the target. Basically it depends on the type of scattering mechanism involved by the target. The self multiplication of coherency vector and its conjugate transpose will help in forming the coherency matrix. The section III describes the mathematical representation of the covariance and coherency matrix.

\section{MATHEMATICAL REPRESENTATION OF COHERENCY MATRIX}

\section{A. Covariance Matrix:}

The basic formation of this matrix is by target vector called as covariance vector denoted as $k_{C}$.

$$
k_{C}=\left[\begin{array}{c}
k_{1} \\
k_{2} \\
k_{3}
\end{array}\right]=\left[\begin{array}{c}
S_{H H} \\
\sqrt{2} S_{H V} \\
S_{V V}
\end{array}\right] \ldots \ldots(1)
$$

The elements $S_{\mathrm{HH}}$ and $\mathrm{S}_{\mathrm{Vv}}$ are the co polarized element. The $\mathrm{S}_{\mathrm{HV}}$ represents the cross polarized element. The reciprocity condition is satisfied by this vector. It indicates equivalence between $\mathrm{S}_{\mathrm{VH}}$ and $\mathrm{S}_{\mathrm{HV}}$. The linear decomposition technique is considered as basic decomposition technique. Here information obtained from individual polarization channel is considered. The information obtained 
corresponds to cross polarized scatter. The three vectors are $\mathrm{k}_{1}, \mathrm{k}_{2}$ and $\mathrm{k}_{3}$. The $\mathrm{k}_{1}$ represents the scatter of horizontal oriented target scattering. The second component $\mathrm{k}_{2}$ is by cross polarized scatter. The third component $\mathrm{k}_{3}$ is scatter of vertical oriented target scattering. The covariance matrix is represented by $\mathrm{C}$. It is obtained by multiplication of $k_{C}$ itself. $\mathrm{T}$ indicates transpose operation on $k_{C}$.

$$
C=k_{C} * k_{C}^{* T}
$$

\section{B. Coherency matrix}

The vector called coherency vector is termed by $k_{P}$. The vectorization is carried out in Pauli spin type. Here also the reciprocity condition is considered.

$$
k_{P}=\left[\begin{array}{c}
k_{1} \\
k_{2} \\
k_{3}
\end{array}\right]=\frac{1}{\sqrt{2}}\left[\begin{array}{c}
S_{H H}+S_{V V} \\
S_{H H}-S_{V V} \\
2 S_{H V}
\end{array}\right] \ldots \ldots \text { (3) }
$$

The vector basically represents three main scattering mechanisms called odd bounce, even bounce and diffuse scattering. Pauli decomposition uses Pauli matrices for the extension of scattering matrix. It is basically applied to deterministic targets of consideration. It consists of four scattering mechanisms. The first is called single scattering from plane surface. The second and third parameter is called diplane scattering. It is also called as double or even bounce scattering. Such scattering will occur from corners with orientation of $0^{\circ}$ and $45^{\circ}$. The fourth element is called anti symmetric component of S matrix. For the monostatic radar this term leads to value zero.

The coherency matrix is represented by $\mathrm{T}_{\text {cohe }}$. It is obtained by self multiplication of $\quad k_{P}$

$$
T_{\text {cohe }}=k_{P} * k_{P}^{* T}
$$

The two important decomposition method of SAR polarimetry called linear decomposition and Pauli decomposition can be obtained by using the two scattering vectors $k_{C}$ and

$$
k_{P}
$$

The next section describes the importance of Eigen value decomposition which is the base for entropy based target identification.

\section{ENTROPY BASED TARGET IDENTIFICATION}

The coherency matrix contains information of the scattering process. It is a $3 * 3$ Hermitian semi definite positive matrix [4]. The matrix is called positive definite if its Hermitian or symmetric part has positive Eigen values. This matrix can be written in the form of Eigen values.

$$
T_{\text {cohe }}=\lambda_{1}\left(e_{1} \cdot e_{1}^{T}\right)+\lambda_{2}\left(e_{2} \cdot e_{2}^{T}\right)+\lambda_{3}\left(e_{3} \cdot e_{3}^{T}\right)
$$

The mathematical equation represents the Eigen value decomposition of coherency matrix. The symbol $\mathrm{e}_{1}, \mathrm{e}_{2}$ and $\mathrm{e}_{3}$ represents Eigen vectors. It involves the three basic scattering mechanisms known as surface, double bounce and volume scattering. The prominent parameters of Eigen value decomposition are scattering entropy $(\mathrm{H})$, scattering Anisotropy (A) and mean scattering angle $(\alpha)$ and $\beta$. The entropy $\mathrm{H}$ characterizes the scattering mechanisms.

$$
\begin{aligned}
& H=-\sum_{i=1}^{3} p_{i} \log _{3} p_{i} \ldots \ldots .(6) \\
& A=\frac{\lambda_{2}-\lambda_{3}}{\lambda_{2}+\lambda_{3}} \ldots \ldots \text { (7) } \\
& \alpha=p_{1} \alpha_{1}+p_{2} \alpha_{2}+p_{3} \alpha_{3}
\end{aligned}
$$

Entropy $\mathrm{H}$ and Anisotropy $\mathrm{A}$ are parameters related to eigenvalues $\lambda_{1}, \lambda_{2}, \lambda_{3}$ which are obtained from coherency matrix. The $\alpha$ and $\beta$ are from Eigen vectors. The angle $\alpha$ describes the surface scattering and angle $\beta$ is double of orientation angle.

Here probability $\mathrm{P}_{\mathrm{i}}$ obtained from Eigen value is given by,

$$
p_{i}=\frac{\lambda_{i}}{\lambda_{1}+\lambda_{2}+\lambda_{3}}
$$

The entropy characterizes the feature of randomness involved through the scattering by the target.

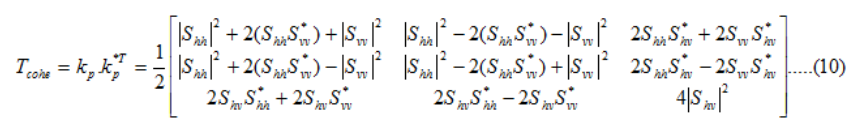

The empirical formula used by the Technical Committee of Canada Centre for remote sensing for coherency matrix is represented in equation (10) [15]. The same formula has been used in the evaluation of the coherency matrix in python system model. The color space aspect of the image data has the consideration for the execution of the formula used.

Here basically $S_{\mathrm{hh}}$ is represented for red color. $S_{\mathrm{hv}}$ is represented for green color and $S_{\mathrm{vv}}$ is represented for blue color [3].

\section{DISCUSSIONS}

The image characterization is basically obtained by polarization state of the electromagnetic wave. The various scattering mechanisms obtained through natural distributed targets basically depend on wavelength, dielectric constant and polarization of the EM wave.

TABLE I. PARAMETERS OF ENTROPY BASED TARGET IDENTIFICATION 


\begin{tabular}{|c|c|c|c|}
\hline \multirow[b]{2}{*}{$\begin{array}{l}\text { Decomposition } \\
\text { Method }\end{array}$} & \multicolumn{3}{|c|}{ Feature Parameters } \\
\hline & $\begin{array}{c}\mathrm{H} \\
\text { Entropy }\end{array}$ & $\begin{array}{c}\text { A } \\
\text { Anisotropy }\end{array}$ & $\begin{array}{c}\alpha \\
\text { Mean Scattering } \\
\text { Angle } \\
\end{array}$ \\
\hline \multirow[t]{2}{*}{$\begin{array}{l}\text { Entropy based } \\
\text { target } \\
\text { identification }\end{array}$} & $\begin{array}{c}\mathrm{H}=0 \\
\text { only one } \\
\text { Eigen value } \\
\text { one } \\
\text { scattering } \\
\text { process } \\
\text { surface } \\
\text { scattering }\end{array}$ & \multirow{2}{*}{$\begin{array}{l}\text { Two Eigen } \\
\text { values }\end{array}$} & \multirow{2}{*}{$\begin{array}{c}\alpha=0^{\circ} \\
\text { surface scattering } \\
\alpha=45^{\circ} \\
\text { dipole scattering } \\
\alpha=90^{\circ} \\
\begin{array}{c}\text { double bounce } \\
\text { scattering }\end{array}\end{array}$} \\
\hline & $\begin{array}{c}\mathrm{H}=1 \\
\text { Three equal } \\
\text { Eigen } \\
\text { values } \\
\text { Three } \\
\text { scattering } \\
\text { process }\end{array}$ & & \\
\hline
\end{tabular}

The mathematical modeling of the target identification is carried by coherency matrix. Identifying the target vector and evaluation of the target vector for entropy based target identification is the major contribution of this paper.

The parameter entropy is the measurement of random feature of scattering process. The degree of randomness of scattering is defined as entropy. Entropy is normally in the range of 0 to unity. It is the logarithmic sum of eigenvalues of coherency matrix.

Anisotropy is the parameter to enhance the quality of the entropy. It gives the relation between scattering which is basically expressed in terms of second and third Eigen values.

TABLE I depicts the parameters of entropy based target identification. The information in the table reflects the scattering property of the target distributed on the earth surface. The backscattering information helps in identifying the target. SAR image is basically combination of energy present for individual image pixel. It depicts the combination of three images. The interpretation of polarization state change of the backscatter is obtained by target vector of the entropy based target identification.

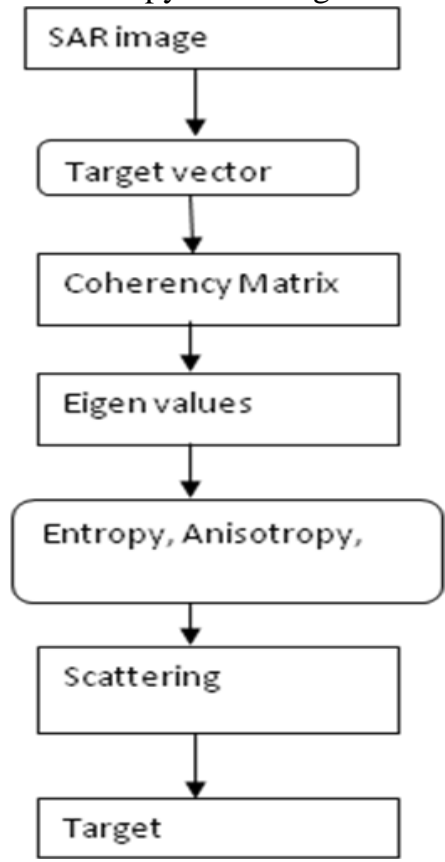

Fig. 1 Entropy based target identification method
Fig. 1 represents the entropy based target identification method adopted for coherency matrix of SAR image. The scattering properties are characterized by scattering matrix. The elements represent the combination of transmit and receive polarization states.

The coherency matrix and its Eigen values are evaluated through image analysis using Python system model. The Entropy values had range between 0 and unity. The Anisotropy values were between -0.5 to 4.7 .

TABLE II. CLASSIFICATION OF ENTROPY

\begin{tabular}{|c|c|c|c|}
\hline \multirow{2}{*}{ Parameter } & \multicolumn{3}{|c|}{ Area } \\
\cline { 2 - 4 } & Zonel & Zone 2 & Zone3 \\
\hline Entropy & Low & Medium & High \\
& $\begin{array}{c}\text { Surface } \\
\text { scattering }\end{array}$ & $\begin{array}{c}\text { Double bounce } \\
\text { Scattering } \\
\text { Secondary } \\
\text { scattering }\end{array}$ & $\begin{array}{c}\text { Multiple } \\
\text { scattering }\end{array}$ \\
$\begin{array}{c}\text { Single } \\
\text { scattering } \\
\text { from volume } \\
\text { scattering }\end{array}$ \\
\hline
\end{tabular}

TABLE II depicts the inference drawn from the obtained data.

The data analysis is carried out for set of three different values of entropy.

Accordingly value has been represented for Low, Medium and High kind of entropy. These three ranges of entropy values do represent three different areas. The characteristic of these areas depicts various scattering mechanisms involved by the target. The high value of entropy basically depicts multiple scattering by volumetric information. The low value of entropy represents the surface scattering mechanism by the single surface. The medium entropy values depicts double bounce scattering. The single bounce scattering basically keeps the direction of polarization orientation same. The double bounce scattering represents a kind of definite scattering. The volume scattering is combination of various behavior of scattering. The value of 0 degree for alpha angle specifies the surface scattering. The values $45^{\circ}$ and $90^{\circ}$ are referred for dipole and double bounce scattering.

The two basic polarization featured vectors $k_{C}$ and $k_{P}$ are used in most of the decomposition theory related to SAR polarimetry data. The various types of matrices are developed to represent the scattering behavior of the target. The various matrices are covariance matrix, coherency matrix, Stokes matrix, Mueller Matrix.

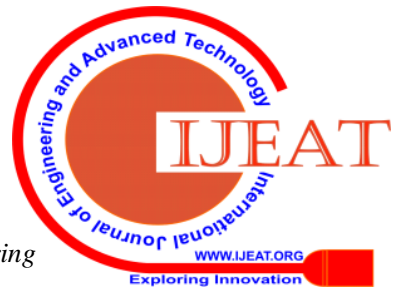


The covariance matrix is formed by the multiplication of lexicographic description and its transpose where as coherency matrix is formed by Pauli based description and its transpose.

\section{CONCLUSIONS}

This paper gives basic ideas regarding the scattering target vector which are helpful for decomposition models. The importance of covariance and coherency matrices are described. The coherency matrix is highlighted and evaluated by the coherency target vector. The effect of scattering mechanism is analyzed by the parameter entropy. System model for entropy based target identification is developed. The involvement of Eigen decomposition techniques for SAR polarimetry is realized. The target vector depicts the scattering property of the backscattering nature of the distributed targets.

\section{REFERENCES}

1. Kazuo Ouchi, "Recent Trend and Advance of Synthetic Aperture Radar with Selected Topics," Review Remote Sensing ISSN 2072 4292 , vol. 1, no. 1, pp. 716-765, Feb. 2013.

2. M.Ouarzeddine, B. Souissi, A. Belhadj-Aissa, "Classification of Polarimetric SAR images based on scattering mechanisms,' University of Science and Technology Houri Boumediene, vol. 1, no. 1, pp.1-6, Jan. 2007.

3. Manuel E. Arrigada, "Performance of scattering matrix decomposition and color space for synthetic aperture radar imagery," Master Thesis for degree of Master of Science, pp. 1-73, Mar. 2010.

4. Maurizio Sarti, Lucio Mascolo, "An investigation of different polarimetric decomposition techniques for soil moisture estimation," IEEE,vol. 1, no. 1, pp. 209-213, Sept. 2012.

5. Shenglong GUO, Yang, Wen HONG, Jianfeng Wang and Xiaoyang Guo, "Model based target decomposition with $\pi / 4$ mode compact polarimetry data," Science China Information Sciences, vol. 59, no. 1, pp.1-10, Jun. 2016.

6. Hongbo Sun,Masanobu Shimada and Feng Xu, "Recent Advances in Synthetic Aperture Radar Remote Sensing-Systems, Data Processing and Applications," IEEE Geoscience Remote Sensing Letter, vol. 14, no. 11, pp. 2013-2016, Nov. 2017.

7. Shashikumar, "Advances in Polarimetry," SPIE Asia Pacific Remote sensing APRS Symposium tutorial, vol. 1, no.1, pp. 1-23, Apr. 2016.

8. Jacob Van Zyl and Yunjin Kim, "Synthetic Aperture Radar Polarimetry," Jet Propulsion Laboratory, California Information Technology, pp. 88-100, Dec. 2010.

9. Si-Wei Chen, Yong-Zhen Li, Xue-Song Wang, Shan-Ping Xiao and Motoyuki Sato, "Modelling and interpretation of scattering mechanism in polarimetric synthetic aperture radar," IEEE signal processing magazine, vol. 1, no. 1, pp. 79-89, Jul. 2014.

10. Sang-Hoon Hong and Shimon Wdowinski, "Revising vegetation scattering theories: Adding a rotated dihedral double bounce scattering to explain cross-polarimetric SAR observations over wetlands," Proceedings, 'Fringe 2011 Workshop', Frascati, Italy, pp. 1-7, Sep. 2011.

11. Eric Pottier, "Recent advances in the development of the open source toolbox for polarimetric and interferometric polarimetric SAR data processing: The POLSARPRO v4.1.5 software," IEEE, vol. 1, no. 1, pp. 2527-2530, Aug. 2010.

12. Jong sen Lee and Thomas L. Ainsworth, "An Overview of Recent Advances In Polarimetric SAR information Extraction: Algorithms and Applications," IEEE International Geoscience and Remote sensing Symposium, pp. 851-854, Jul. 2010.

13. Alberto Moreira, Pau Prats-Iraola, Marwan Younis, Gerhard Krieger,Iren Hajnsek and Konstantinos P. Papathanassiou,Microwave and Radar institute of the German Aerospace Center (DLR), Germany, "A Tutorial on Synthetic Aperture Radar," IEEE Geoscience and remote sensing magazine, pp. 6-38, Mar. 2013.

14. C.P. Schwegmann, W. Kleynhans, B. P. Salmon, "The Development of Deep learning in Synthetic Aperture Radar Imagery," International workshop on remote sensing with Intelligent Processing, vol.1, no.1, pp.1-6, May. 2017.

15. Technical Committee, "Advanced Radar Polarimetry Tutorial-Radar polarimetry," Technical report, Canada centre for remote sensing, pp. 1-24, Nov. 2015.
16. Yonglei Chang, Jie Yang, Pingxiang Li , Lingli Zhao, Lei Shi "Sample extraction based on helix scattering for polarimetric sar calibration," The International Archives of the Photogrammetry, Remote Sensing and Spatial Information Sciences, vol. XLII-2/W7, pp. 1-4, Sep. 2017.

17. Sverre Holm, "Synthetic Aperture Radar and Sonar-SAR and SAS," Lecture notes, Department of informatics, University of OSLO, pp.119, May. 2015.

18. Yoshio Yamaguchi, Toshifumi Moriyama, Motoi Ishido, and Hiroyoshi Yamada, "Four-Component Scattering Model for Polarimetric SAR Image Decomposition," IEEE Transactions on Geoscience and remote sensing, vol. 43, no. 8, Aug. 2005.

19. Yoshio Yamaguchi, Akinobu Sato, Wolfgang-Martin Boerner, Ryoichi and Hiroyoshi Yamada, "Four-Component Scattering Power Decomposition With Rotation of Coherency Matrix," IEEE Transactions on Geoscience and Remote sensing, vol. 49, no. 6, Jun. 2011.

20. Shane Robert Cloude and Eric Pottier, "A review of target decomposition theorems in radar polarimetry," IEEE Transactions on Geoscience and Remote sensing, vol. 34, no. 2, pp. 1-4, Mar. 1996.

21. Thuy LeToan , "SAR Image classification content scattering physics," ESA most dragon programme Advanced training course in Land Remote sensing, Beijing, pp. 1-4, Oct. 2005.

22. Muhtar Qong, Takeo Tadono, Hiroyuki Wakabayashi and Masanobu Shimada, "Muller Matrix Based Classification of Polarimetric SAR Data," Earth Observation Research Center , National Space Development Agency of Japan, IEEE, vol. 1, no. 1, pp. 375-377, Dec. 2000.

23. Kohei Arai , " Polarimetric SAR Image Classification with High Frequency Component Derived from Wavelet Multi Resolution Analysis:MRA," International Journal of Advanced Computer Science and Applications(IJACSA), vol. 2, no. 9, pp. 37-42, Dec. 2011.

24. Camilla Brekke, Cathleen E. Jones, Stine Skrunes, Benjamin Holt, Martine Espeseth and Torbjorn Eltoft, " Cross Correlation between Polarization Channels in SAR Imagery over Oceanographic Features," IEEE Geoscience and remote sensing letters. vol. 1, no. 1, pp. 1-5, Jan. 2011.

\section{AUTHORS PROFILE}

Prof. Plasin Francis Dias Received B.E. degree in Electronics and

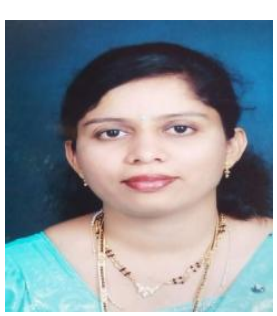

Communication Engineering from Rura Engineering College, Karnatak University Dharwad, India in 1998 and M. Tech in VLSI Design and Embedded Systems from B.V.B. College of Engineering and Technology (BVBCET), Hubli under Visvesvaraya Technological University (VTU), Karnataka, India in 2009. She is Research scholar at BVBCET, Hubli and presently working as Assistant Professor at KLS VDIT, Haliyal, Karnataka. She is the member of ISTE and IEI. Her current areas of research include Signal processing Synthetic Aperture Radar, VLSI design and Embedded System. She has presented four research papers at International Conferences. She has won the Best Paper Award for the paper titled "Entropy Based target Identification in Synthetic Aperture Radar Polarimetry," in the $2^{\text {nd }}$ World Summit on Advances in Science, Engineering and Technology, Indiana Summit 2019 , at Indiana University Perdue University Indianapolis, USA on $3-5^{\text {th }}$ October 2019 organized by Vellore Institute of Technology(VIT), India. She has visited two countries Australia and USA.

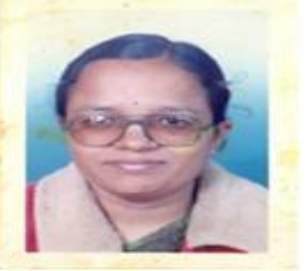

Dr. Rajeshwari M. Banakar Received B.E. degree in Electronics and Communication Received B.E. degree in Electronics and Communication Engineering from Karnatak University India in 1984 and M. Tech in Digita Communication from Regional Engineering College, Surathkal, Karnataka. She has a couple of years

experience in Indian Space Research Organization (ISRO). She completed her Ph.D. in the area of Low Power Application Specific Design Methodology from IIT Delhi in 2004.

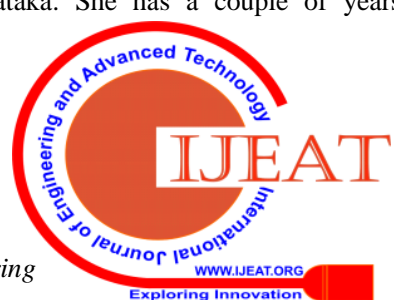




\section{Entropy Based Target Identification in Synthetic Aperture Radar Polarimetry}

Presently she is working as Professor in BVB Engineering College Hubli Karnataka. She is the member of ISTE, IETE, MIE and IEEE scientific and professional societies.

Prof. Banakar's work on Low power scratch pad memory organization presented at CODES 2002 has been rated as highly cited work in short period of four years (2002-2006). She has won the best paper award in 2004 at International conference - MobiComNet, India. She has also contributed to a chapter on Turbo Equalizer in Handbook of Research in Mobile Business (Australia), 2006. In Cadence design contest 2009, she is the recipient of runner up award. Her current areas of research include SOC, VLSI Architecture, WCDMA and Adhoc networks. She has published over 55 research papers. 